Pacific Journal of Mathematics

BOUNDS FOR NUMBERS OF GENERATORS OF 


\title{
BOUNDS FOR NUMBERS OF GENERATORS OF COHEN-MACAULAY IDEALS
}

\author{
JUDith D. SAlLY
}

Let $(R, \underline{m})$ be a local Cohen-Macaulay ring of dimension $d$ and multiplicity $e(R)=e$. A natural question to ask about an $\underline{m}$-primary ideal $I$ is whether there is any relation between the number of generators of $I$ and the least power $t$ of $\underline{m}$ contained in $I$. ( $t$ will be called the nilpotency degree of $R / I)$ It is quite straight forward to obtain a bound for $v(I)$, the number of generators in a minimal basis of $I$, in terms of $t$ and $e$. However, there are several interesting applications. The first is the existence of a bound for the number of generators of any Cohen-Macaulay ideal $I$, i.e. any ideal $I$ such that $R / I$ is Cohen-Macaulay, in terms of $e(R / I), e(R)$ and height $I$. The second application is a bound in terms of $d$ and $e$ for the reduction exponent of $\underline{m}$.

1. $m$-primary ideals. In this section we will use only the standard facts about the existence and properties of superficial elements. However, later we will need a result stronger than the usual existence theorem for these elements so we take this opportunity to recall the definition and prove this special form of the existence theorem.

Definition. Let $(R, \underline{m})$ be a local ring. An element $x$ in $\underline{m}$ is superficial for $\underline{m}$ if there is an integer $c>0$ such that

$$
\left(\underline{m}^{n}: x\right) \cap \underline{m}^{c}=\underline{m}^{n-1} \text { for all } n>c \text {. }
$$

It is a standard fact that $x$ is superficial for $\underline{m}$ if and only if there is an integer $c>0$ such that $0 \neq \bar{X} \in \underline{m} / \underline{m}^{2}=G_{1}$ and

$$
(0: \bar{x} G) \cap G_{n}=0
$$

for $n \geqq c$ where $G_{n}=m^{n} / m^{n+1}$, and $G=G_{0} \oplus G_{1} \oplus \cdots$.

LEMMA 1.1. Let $(R, \underline{m})$ be a local ring with $R / \underline{m}$ infinite. Let $I, J_{1}, \cdots, J_{s}$ be distinct ideals of $R$ which are also distinct from $\underline{m}$. Then there is an element $x$ in $R$ such that

(1) $x \notin J_{i}$,

(2) $x$ is a superficial element for $\underline{m}$ and $i=1, \cdots, s$

(3) the image of $x$ in a superficial element for $\underline{m} / I$.

Proof. $\quad$ Let $G=R / \underline{m} \oplus \underline{m} / \underline{m}^{2} \oplus \cdots$ and $\bar{G}=R / \underline{m} \oplus \underline{m} / \underline{m}^{2}+I \oplus$ 
$\underline{m}^{2}+I / \underline{m}^{3}+I \oplus \cdots$. Then $\bar{G}=G / K$ where $K$ is a homogeneous ideal of $G$. Let Ass $G=\left\{P_{1}, \cdots P_{t}\right\}$, Ass $\bar{G}=\left\{Q_{1} / K, \cdots, Q_{l} / K\right\}$, where the $Q_{i}$ are primes in $G$. Suppose that $P_{t}=\underline{m} / \underline{m}^{2} \oplus \underline{m}^{2} / \underline{m}^{3} \oplus \cdots$ and $Q_{l}=P_{t} / K$. The following subspaces are all proper $R / \underline{m}$-subspaces of $\underline{m} / \underline{m}^{2}$ :

$$
\begin{aligned}
& J_{i}+\underline{m}^{2} / \underline{m}^{2}, i=1, \cdots, n ; P_{i} \cap\left(\underline{m} / \underline{m}^{2}\right), \quad i=1, \cdots, t-1 \\
& Q_{i} \cap\left(\underline{m} / \underline{m}^{2}\right), i=1, \cdots, l-1 ; I+\underline{m}^{2} / \underline{m}^{2} .
\end{aligned}
$$

Since $R / \underline{m}$ is infinite there is a nonzero $\bar{x}$ in $\underline{m} / \underline{m}^{2}$ such that $\bar{x}$ is not in any of these subspaces. We claim that if $x$ is any element of $\underline{m}$ which maps to $\bar{x}$, then $x$ is superficial for $\underline{m}$ and the image of $x$ in $R / I$ is superficial for $\underline{m} / I$. We need $(0: G \bar{x}) \cap \underline{m}^{n} / \underline{m}^{n+1}=0$ and $(0: \bar{G}(x+I / I)) \cap \underline{m}^{n}+I / \underline{m}^{n+1}+I=0$ for large $n$. Let $\overline{0}=N_{1} \cap$ $\cdots \cap N_{t-1} \cap N_{t}$ with $N_{i} P_{i}$-primary be a primary decomposition of 0 in $G$. Then $(0: G \bar{x}) \subseteq N_{1} \cap \cdots \cap N_{t-1}$. But $\left(\underline{m} / \underline{m}^{2}\right)^{c} \subseteq N_{t}$ for some $c$, hence $(0: G \bar{x}) \cap \underline{m}^{n} / \underline{m}^{n+1}=0$ for $n \geqq c$. The same reasoning shows that the image of $x$ in $R / I$ is a superficial element for $\underline{m} / I$.

Theorem 1.2. Let $(R, \underline{m})$ be a Cohen-Macaulay local ring of dimension $d>0$. Let be an $\underline{m}$-primary ideal and $t$ the nilpotency degree of $R / I$. Then

$$
v(I) \leqq t^{d-1} e(R)+d-1
$$

Proof. The proof is by induction on $d$. If $d=1$, the theorem is well-known (cf. [6] or [7]) but we include a proof for completeness. We may assume that $R / \underline{m}$ is infinite so that $\underline{m}$ has a superficial element $x$ which is also a nonzero divisor. Since $d=1, x \underline{m}^{n}=\underline{m}^{n+1}$ for some $n>0$. We have $\lambda(R / x R)=\lambda(I / x I)=e(R)$, where $\lambda(B)$ denotes the length of an $R$-module $B$. The exact sequence

$$
0 \longrightarrow \underline{m} I / x I \longrightarrow I / x I \longrightarrow I / \underline{m} I \longrightarrow 0
$$

gives $\lambda(I / \underline{m} I)=\lambda(I / x I)-\lambda(\underline{m} I / x I)=e(R)-\lambda(\underline{m} I / x I)$.

Assume $d>1$. Again assuming that $R / \underline{m}$ is infinite as we may, there is a nonzero divisor $x$ such that $x$ is a superficial element for $\underline{m}$. Pass to the $d-1$ dimensional Cohen-Macaulay ring $R / x^{t} . \quad I / x^{t}$ is $\underline{m} / x^{t}$-primary so, by induction,

$$
v\left(I / x^{t}\right) \leqq t^{d-2} e\left(R / x^{t}\right)+d-2 .
$$

Hence $v(I) \leqq v\left(I / x^{t}\right)+1 \leqq t^{d-2} t e(R)+d-1$.

REMARKS. 1. If $(R, \underline{m})$ is regular local and $d \geqq 2$ then $v(I) \leqq$ $g t^{d-2}+d-1$, where $g$ is the degree of $I$, i.e. $I \subseteq \underline{m}^{g} \backslash \underline{m}^{g+1}$.

2. (1.2) generalizes a result of Abhyankar [1]. In [1], Abhyankar 
shows that the Cohen-Macaulay hypothesis is necessary.

If $(R, \underline{m})$ is a $d$-dimensional local ring, the Hilbert function $H$ of $\underline{m}$ is defined as follows:

$$
H(n)=v\left(\underline{m}^{n}\right)=\lambda\left(\underline{m}^{n} / \underline{m}^{n+1}\right)
$$

for integers $n \geqq 0$. For large $n, H(n)$ is a polynomial of degree $d-1$. If we apply (1.2) to $I=\underline{m}^{n}$ we obtain, for Cohen-Macaulay rings, a polynomial of degree $d-1$ which bounds $H(n)$ for all $n$.

Corollary 1.3. Let $(R, \underline{m})$ be a Cohen-Macaulay local ring of multiplicity $e$ and dimension $d>0$. Then, if $P(n)$ is the polynomial, $P(n)=e n^{d-1}+d-1, H(n) \leqq P(n)$ for all $n>0$.

Using a trick of Kirby [4] we have

CoRollary 1.4. Let $I$ be an ideal ideal of a d-dimensional Cohen-Macaulay local ring $(R, \underline{m}), d>0$. Define the Artin-Rees number of $I, a(I)$, to be the least integer a such that $I \cap \underline{m}^{a} \cong I \underline{m}$. Then

$$
v(I) \leqq a^{d-1} e(R)+d-1 .
$$

Proof. $I / I \underline{m}=I / I \underline{m}+I \cap \underline{m}^{a}=I / I \cap I \underline{m}+\underline{m}^{a} \cong I+\underline{m}^{a} / I \underline{m}+\underline{m}^{a}$. Hence $v(I) \leqq v\left(I+\underline{m}^{a}\right)=\lambda\left(I+\underline{m}^{a} / \underline{m}\left(I+\underline{m}^{a}\right)\right)$. So by $(1.2), v(I) \leqq$ $\alpha^{d-1} e(R)+d-1$.

Note that if $R / I$ is Cohen-Macaulay, then $a(I) \leqq e(R / I)+1$ but in general $a(I)$ is not bounded by $e(R / I)$.

2. Applications. If $(R, \underline{m})$ is a local ring, an ideal $I$ is a Cohen-Macaulay ideal if $R / I$ is a Cohen-Macaulay ring.

Theorem 2.1. Let $(R, \underline{m})$ be a d-dimensional Cohen-Macaulay local ring. Let $I$ be a Cohen-Macaulay ideal of height $h>0$. Then

$$
v(I) \leqq e(R / I)^{h-1} e(R)+h-1 .
$$

Proof. We may assume $R / \underline{m}$ is infinite. The proof is by induction on $s=\operatorname{dim} R / I$. If $s=0$ then by (1.2) it is sufficient to note that $e(R / I)=\lambda(R / I) \geqq$ nilpotency degree of $R / I$.

Assume $s>0$. By (1.1) there is a nonzero divisor $x$ in $\underline{m}$ such that $x$ is superficial for $\underline{m}$, and the image of $x$ in $R / I$ is a nonzero divisor in $R / I$ and is a superficial element for $\underline{m} / I$. We pass to the $d-1$ dimensional Cohen-Macaulay ring $R / x$ and to the height $h$ Cohen-Macaulay ideal $(I, x) / x$. By induction $v(I)=v((I, x) / x) \leqq$ $e(R /(I, x))^{h-1} e(R / x)+h-1=e(R / I)^{h-1} e(R)+h-1$. 
REMARKS 1. For height 1 ideals $I,(2.1)$ gives Rees' theorem [6] stating that $v(I) \leqq e(R)$. If height $I=2$, Rees [6] has the result $v(I) \leqq e(R)+e(R / I)$ which gives a better bound than (2.1) except when $R$ or $R / I$ is regular.

2. If $R$ is an equicharacteristic regular local ring, Becker [2] has results similar to (2.1).

Another application of (1.2) gives a bound for what we will call the reduction exponent $r(\underline{m})$ of $\underline{m}$, where $\underline{m}$ is the maximal ideal of a $d$-dimensional Cahen-Macaulay local $\operatorname{ring}(R, \underline{m})$. Assume that $R / \underline{m}$ is infinite. $r(\underline{m})$ is the least integer $r$ such that there exists a system of parameters $\bar{x}_{1}, \cdots, \bar{x}_{d}$ of degree 1 in $G=R / \underline{m} \oplus \underline{m} / \underline{m}^{2} \oplus \cdots$ with $\mathscr{C}^{r} \subseteq\left(\bar{x}_{1}, \cdots, \bar{x}_{d}\right)$ where $\mathscr{C l}=\underline{m} / \underline{m}^{2} \oplus \underline{m}^{2} / \underline{m}^{3} \oplus \cdots$.

Theorem 2.2. Let $(R, \underline{m})$ be a d-dimensional local Cohen-Macaulay ring of multiplicity $e(R)$ with $R / \underline{m}$ infinite and $d>0$. Then

$$
r(\underline{m}) \leqq d ! e(R)-1 \text {. }
$$

Proof. In our case the main theorem of Eakin and Sathaye in [3] states that $H(n)<\left(\begin{array}{c}n+d \\ d\end{array}\right)$ implies $r(\underline{m}) \leqq n$. By Corollary 1.3, $H(n) \leqq n^{d-1} e(R)+d-1$. So it is sufficient to note that

$$
(d ! e(R))^{d-1} e(R)+d-1<\left(\begin{array}{c}
d ! e(R)+d \\
d
\end{array}\right) .
$$

This generalizes a result in [7] where $R$ was assumed to be of dimension 1.

\section{REFERENCES}

1. S. Abhyankar, Local rings of high embedding dimension, A. J. M., 89 (1967), 10731077.

2. J. Becker, On the boundedness and unboundedness of the number of generators of ideals and multiplicity, preprint.

3. P. Eakin and A. Sathaye, Prestable ideals, to appear in J. of Algebra.

4. D. Kirby, The reduction number of a one dimensional local ring, J. London Math. Soc., (2) 10 (1975), 471-481.

5. D. Northcott and D. Rees, Reductions of ideals in local rings, Proc. Camb. Phil. Soc., 50 (1954), 145-158.

6. D. Rees, Estimates for the minimum number of generators of modules over CohenMacaulay local rings, preprint.

7. J. Sally and W. Vasconcelos, Stable rings, J. of Pure and Appl. Algebra, 4 (1974), 319-336.

Received December 1, 1975.

NORTHWESTERN UNIVERSITY 


\title{
PACIFIC JOURNAL OF MATHEMATICS
}

\author{
EDITORS
}

\author{
RICHARD ARENS (Managing Editor) \\ University of California \\ Los Angeles, California 90024
}

\author{
R. A. Beaumont \\ University of Washington \\ Seattle, Washington 98105
}

J. DugundjI

Department of Mathematics

University of Southern California

Los Angeles, California 90007

D. Gilbarg and J. Milgram

Stanford University

Stanford, California 94305

\section{ASSOCIATE EDITORS}
E. F. BECKENBACH
B. H. NEUMANN
F. WOLF
K. Yoshida

\section{SUPPORTING INSTITUTIONS}

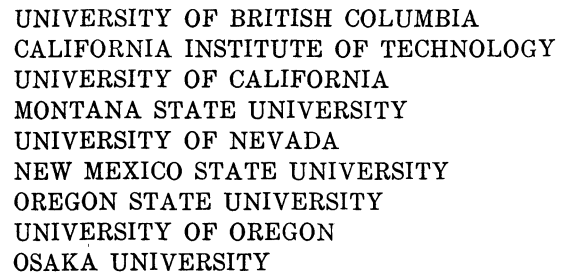

UNIVERSITY OF BRITISH COLUMBIA CALIFORNIA INSTITUTE OF TECHNOLOGY UNIVERSITY OF CALIFORNIA MONTANA STATE UNIVERSITY UNIVERSITY OF NEVADA NEW MEXICO STATE UNIVERSITY OREGON STATE UNIVERSITY UNIVERSITY OF OREGON OSAKA UNIVERSITY

\author{
UNIVERSITY OF SOUTHERN CALIFORNIA \\ STANFORD UNIVERSITY \\ UNIVERSITY OF HAWAII \\ UNIVERSITY OF TOKYO \\ UNIVERSITY OF UTAH \\ WASHINGTON STATE UNIVERSITY \\ UNIVERSITY OF WASHINGTON

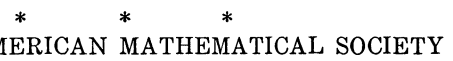

The Supporting Institutions listed above contribute to the cost of publication of this Journal, but they are not owners or publishers and have no responsibility for its content or policies.

Mathematical papers intended for publication in the Pacific Journal of Mathematics should be in typed form or offset-reproduced, (not dittoed), double spaced with large margins. Please do not use built up fractions in the text of your manuscript. You may however, use them in the displayed equations. Underline Greek letters in red, German in green, and script in blue. The first paragraph or two must be capable of being used separately as a synopsis of the entire paper. Items of the bibliography should not be cited there unless absolutely necessary, in which case they must be identified by author and Journal, rather than by item number. Manuscripts, in triplicate, may be sent to any one of the editors. Please classify according to the scheme of Math. Reviews, Index to Vol. 39. All other communications should be addressed to the managing editor, or Elaine Barth, University of California, Los Angeles, California, 90024.

The Pacific Journal of Mathematics expects the author's institution to pay page charges, and reserves the right to delay publication for nonpayment of charges in case of financial emergency.

100 reprints are provided free for each article, only if page charges have been substantially paid. Additional copies may be obtained at cost in multiples of 50 .

The Pacific Journal of Mathematics is issued monthly as of January 1966. Regular subscription rate: $\$ 72.00$ a year (6 Vols., 12 issues). Special rate: $\$ 36.00$ a year to individual members of supporting institutions.

Subscriptions, orders for back numbers, and changes of address should be sent to Pacific Journal of Mathematics, 103 Highland Boulevard, Berkeley, California, 94708.

PUBLISHED BY PACIFIC JOURNAL OF MATHEMATICS, A NON-PROFIT CORPORATION

Printed at Kokusai Bunken Insatsusha (International Academic Printing Co., Ltd.), 8-8, 3-chome, Takadanobaba, Shinjuku-ku, Tokyo 160, Japan.

Copyright (C) 1975 by Pacific Journal of Mathematics Manufactured and first issued in Japan 


\section{Pacific Journal of Mathematics}

\section{Vol. 63, No. 2 \\ April, 1976}

Joseph Anthony Ball and Arthur R. Lubin, On a class of contractive perturbations

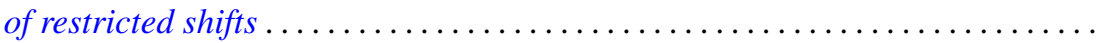

Joseph Becker and William C. Brown, On extending higher derivations generated

by cup products to the integral closure .......................

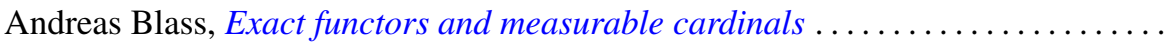

Joseph Eugene Collison, A variance property for arithmetic functions . . . . . . . . . .

Craig McCormack Cordes, Quadratic forms over nonformally real fields with a

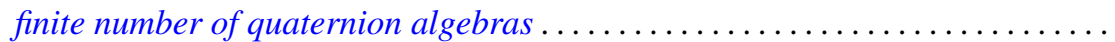

Freddy Delbaen, Weakly compact sets in $H^{1} \ldots \ldots \ldots \ldots \ldots \ldots \ldots \ldots \ldots$

G. D. Dikshit, Absolute Nörlund summability factors for Fourier series ..........

Edward Richard Fadell, Nielsen numbers as a homotopy type invariant. . ........

Josip Globevnik, Analytic extensions of vector-valued functions . . . . . . . . . . . .

Robert Gold, Genera in normal extensions . . . . . . . . . . . . . . . . . . . 389

Solomon Wolf Golomb, Formulas for the next prime

Robert L. Griess, Jr., The splitting of extensions of $S L(3,3)$ by the vector space

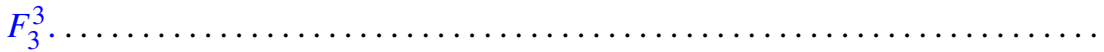

Thomas Alan Keagy, Matrix transformations and absolute summability .........

Kazuo Kishi, Analytic maps of the open unit disk onto a Gleason part.

Kwangil Koh, Jiang Luh and Mohan S. Putcha, On the associativity and commutativity of algebras over commutative rings . ..... . .

James C. Lillo, Asymptotic behavior of solutions of retarded differential difference equations.

John Alan MacBain, Local and global bifurcation from normal eigenvalues ..

Anna Maria Mantero, Sets of uniqueness and multiplicity for $L^{p}$

J. F. McClendon, Embedding metric families

L. Robbiano and Giuseppe Valla, Primary powers of a prime ideal .

Wolfgang Ruess, Generalized inductive limit topologies and barrelledness

properties.

Judith D. Sally, Bounds for numbers of generators of Cohen-Macaulay ideals

Helga Schirmer, Mappings of polyhedra with prescribed fixed points and fixed point indices.

Cho Wei Sit, Quotients of complete multipartite graphs

S. Sznajder and Zbigniew Zielezny, Solvability of convolution equations in $\mathscr{K}_{p}^{\prime}$,

$p>1$.

Mitchell Herbert Taibleson, The existence of natural field structures for finite

dimensional vector spaces over local fields

William Yslas Vélez, A characterization of completely regular fields

P. S. Venkatesan, On right unipotent semigroups ..............

Kenneth S. Williams, A rational octic reciprocity law ............

Robert Ross Wilson, Lattice orderings on the real field .......... 